\title{
Dynamics of coupled multimode and hybrid optomechanical systems
}

\section{Dynamique de systèmes optomécaniques hybrides et multimodes}

\author{
Georg Heinrich $^{\mathrm{a}, *}$, Max Ludwig a, Huaizhi Wu ${ }^{\mathrm{a}, \mathrm{b}}, \mathrm{K}^{\mathrm{a}}$. Hammerer ${ }^{\mathrm{c}}$, Florian Marquardt ${ }^{\mathrm{a}, \mathrm{d}}$ \\ a Institute for Theoretical Physics, University Erlangen-Nürnberg, Staudtstr. 7, 91058 Erlangen, Germany \\ b Department of Physics, Fuzhou University, Fuzhou 350002, PR China \\ c Institute for Theoretical Physics, Institute for Gravitational Physics, Callinstrasse 38, 30167 Hannover, Germany \\ d Max Planck Institute for the Science of Light, Günter-Scharowsky-Str. 1/Bau 24, 91058 Erlangen, Germany
}

\section{A R T I C L E I N F O}

\section{Article history:}

Received 7 June 2010

Accepted after revision 7 February 2011

Available online 9 March 2011

\section{Keywords:}

Multimode optomechanics

Coupled dynamics

Hybrid systems

Mechanically driven coherent photon

dynamics

Cavity-mediated coupling

\section{Mots-clés:}

Optomécanique multimode

Systèmes hybrides

Dynamique cohérente de photons pilotée

par une force mécanique

\begin{abstract}
A B S T R A C T
Recent experimental developments have brought into focus optomechanical systems containing multiple optical and mechanical modes interacting with each other. Examples include a setup with a movable membrane between two end-mirrors and "optomechanical crystal" devices that support localized optical and mechanical modes in a photonic crystal type structure. We discuss how mechanical driving of such structures results in coherent photon transfer between optical modes, and how the physics of Landau-ZenerStueckelberg oscillations arises in this context. Another area where multiple modes are involved are hybrid systems. There, we review the recent proposal of a single atom whose mechanical motion is coupled to a membrane via the light field. This is a special case of the general principle of cavity-mediated mechanical coupling. Such a setup would allow the well-developed tools of atomic physics to be employed to access the quantum state of the 'macroscopic' mechanical mode of the membrane.
\end{abstract}

(C) 2011 Académie des sciences. Published by Elsevier Masson SAS. All rights reserved.

\section{R É S U M É}

Des développements expérimentaux récents ont permis de réaliser des systèmes optomécaniques avec plusieurs modes optiques et mécaniques en interaction. Parmi ceux$\mathrm{ci}$, on trouve par exemple les membranes mobiles placées dans des cavités optiques, et les "cristaux optomécaniques" basés sur des structures à cristaux photoniques dans lesquels les modes optiques et mécaniques sont spatialement confinés. Nous montrons comment l'application d'une force mécanique périodique conduit à un transfert cohérent de photons entre modes optiques, induisant des interférences «Landau-Zener-Stückelberg ". Les systèmes quantiques hybrides constituent un autre domaine où l'interaction entre plusieurs modes est importante. Nous décrivons la proposition récente où un atome unique interagit avec une membrane par l'intermédiaire de la lumière dans une cavité optique. Un tel système permettrait d'utiliser les outils bien maitrisés de la physique atomique pour manipuler l'état quantique du mouvement de la membrane.

(c) 2011 Académie des sciences. Published by Elsevier Masson SAS. All rights reserved.

\footnotetext{
* Corresponding author.

E-mail address: georg.heinrich@physik.Imu.de (G. Heinrich).
} 


\section{Introduction}

Over the past six years, cavity optomechanics has evolved into a research area of its own, with vigorous experimental and theoretical progress at the intersection between nanophysics and quantum optics (for recent reviews see [1] and [2]). The interaction between light and mechanical motion can be most easily understood in the paradigmatic setup of a laser-driven optical cavity with a movable end mirror. The radiation pressure force moves the mirror, and any mirror displacement in turn acts back on the circulating light intensity due to the change in cavity length. The finite photon lifetime induces a time-delay in the radiation pressure force, giving rise to what is known as "dynamical back action". This leads to effects such as a change in the effective mechanical frequency ("optical spring effect"), extra damping and thereby optomechanical cooling, and self-induced optomechanical oscillations. The range of experimental setups in which these basic features have by now been demonstrated is large and growing steadily. It includes mirrors attached to AFM cantilevers and nanobeams [3-6], suspended gram-scale mirrors [7], toroidal micro-cavities [8,9], nanobeams evanescently coupled to the light field of a toroid [10], and many others. There are also setups that do away with the optical radiation field and instead employ microwave radiation traveling in a superconducting transmission line resonator on a chip [11,12], or those that replace the movable mirror by the center-of-mass motion of a cloud of cold atoms [13,14].

Even though any mechanical structure has a multitude of mechanical normal modes and any optical cavity has a discrete spectrum of optical modes, the attention has usually been on just one optical mode interacting with one mechanical mode. The optical mode is selected by the frequency of the incoming laser radiation and the mechanical mode is determined by the mechanical frequency which one chooses to look at in the RF displacement spectrum and which is addressed for cooling experiments by adjustment of the detuning between laser and optical mode. Until quite recently, the physics of multiple modes did not play an important role in experiments, with only a few notable exceptions (e.g. multiple mechanical modes observed in the nonlinear dynamics of self-induced oscillations [15]).

This has now changed with the advent of devices that are deliberately designed to employ the richer flexibility and new functionalities that can be realized in multimode setups.

One example is the "membrane-in-the-middle" setup realized in the Harris lab at Yale [16,17]. The crucial element is a membrane sitting between two fixed end-mirrors. In the limit of a perfectly reflecting membrane, the overall cavity is thus partitioned into two cavities, each of which has their own spectrum of optical resonances. When two of those are close to each other in frequency, the remaining amplitude for photon transmission through the membrane couples these resonances. In this way, hybridized optical modes are formed, and the dependence of the optical mode frequencies on membrane displacement becomes more interesting than the simple linear relation found in the standard setups. The resulting quadratic dependence of optical resonance frequency on displacement could potentially be used to observe quantum jumps between mechanical Fock states in a future optimized device [16-19], or to measure "phonon shot noise" [20]. These functionalities rely crucially on the presence of two coupled optical modes. Adding multiple membranes and/or making use of transverse optical modes [18] could add even more design flexibility. Coupled multimode setups can also increase the sensitivity of displacement measurements [21,22] and enable to implement phonon lasing [23].

Another, even more recent experimental setup which promises better scalability are the "optomechanical crystals" introduced by the lab of O. Painter [24-27]. There, a photonic crystal structure (i.e. a periodically patterned dielectric beam) is suspended freely. Defects are introduced deliberately by varying the spacing of holes in the beam. As a result, both localized optical and mechanical modes are formed, and they interact with each other via the conventional optomechanical interaction. The tight confinement of both types of modes has at least two beneficial aspects. Firstly, the mechanical frequencies go up and can venture into the $\mathrm{GHz}$ regime, which makes it straightforward to cool them into the quantum ground state, in contrast to the lower-frequency setups conventionally employed, which need substantial extra optomechanical cooling. Secondly, the optical frequency pull per displacement, i.e. the optomechanical coupling strength, is enhanced significantly, reaching record values. In addition, the fact that these structures are fabricated using the tools of nanofabrication out of silicon on a chip makes them a perfect candidate for scalable integrated optomechanical devices (as are the structures fabricated in the Tang group at Yale [28]). It also means that it will be relatively straightforward to produce what one may term "optomechanical arrays", where one has multiple optical and mechanical modes interacting with each other.

In the present contribution, we review and elaborate on two of our recent theoretical developments that bear on the possibilities afforded by multimode optomechanical structures. One question which we address is how photons can be deliberately "shuffled around" between different optical modes, in a coherent fashion. We have shown [29] that it is possible to do so via mechanical driving that can induce, for example, photonic Rabi oscillations between optical modes. More generally, one may think of all the multi-level atomic physics (like $\Lambda$ and $V$-type systems), replacing the laser and the atomic levels by the mechanical driving and the optical modes, respectively. Going a bit deeper into the analysis of what happens to a photonic two-level system under an oscillatory drive, one may realize the physics of Landau-Zener-Stückelberg oscillations, with intricate interference patterns showing up in the optical transmission as a function of laser drive frequency, mechanical oscillation amplitude and other parameters.

As another example of multimode physics, we discuss a hybrid setup that was proposed recently [30,31]. It shows how one may employ the optomechanical interaction to induce a coupling between the mechanical motion of a single trapped neutral atom and the vibration of a membrane. The idea is that this may be used to employ the well-developed toolbox of atomic manipulation to indirectly address the motion of the membrane. State transfer and atom-membrane entanglement are just one of the two most elementary example of the possibilities opened up by such a setup. 
(a)

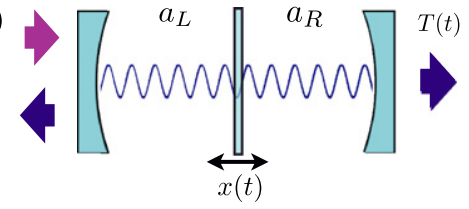

(c)

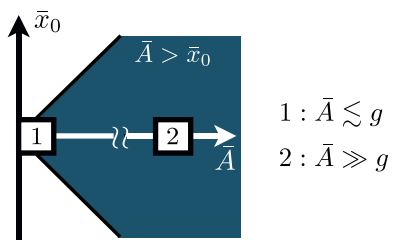

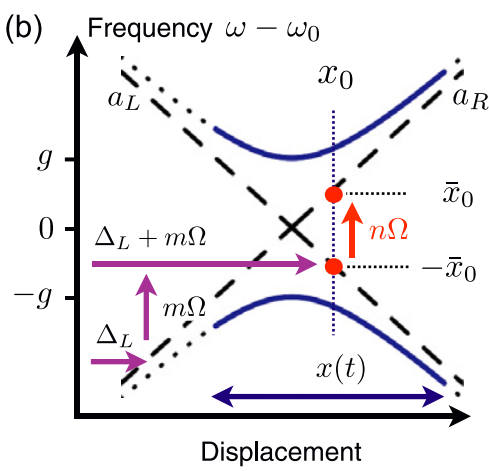

Fig. 1. (a) Setup: a movable, dielectric membrane couples two optical modes $a_{L}, a_{R}$ inside a high-finesse cavity. The left hand side of the cavity is driven by a laser at frequency $\omega_{L}$ while the transmission to the right is recorded. (b) Optical resonance frequency as function of static displacement and multiphonon transition picture: the membrane's displacement linearly changes the modes' frequency (dashed). Due to the coupling there is an avoided-crossing of size $2 \mathrm{~g}$ (dotted). We consider an external driving of the membrane $x(t)=x_{0}+A \cos (\Omega t)$. To see transmission two multiphonon transitions are involved. First, excitation of the left cavity mode at average frequency $-\bar{x}_{0}$, Eq. (3). Second, transferring photons from the left into the right mode, Eq. (4). (c) Overview of parameter regimes for amplitude $\bar{A}=\left(\omega_{0} / l\right) A$ and mean position $\bar{x}_{0}=\left(\omega_{0} / l\right) x_{0}$. For $\bar{A}>\bar{x}_{0}$ (colored), we sweep through the anti-crossing. We distinguish the weak/modest (region 1, see Fig. 2(a)-(b)) and strong (region 2, see Fig. 2(c) and Fig. 3) driving regime.

\section{Mechanically driven coherent photon dynamics}

The standard approach to optomechanics is to characterize mechanical motion under the influence of the light field in steady state. Going beyond this scheme we can ask what happens when the mechanics is exposed to external (periodic) driving. It turns out that the result is most interesting for coupled multimode optomechanical systems that comprise several optical (and vibrational) modes. Here we review the concept of mechanically driven coherent photon dynamics [29] that in principle allows to realize all kinds of driven two- and multilevel dynamics, known for atomic levels from quantum optics, but now in terms of the optical modes in optomechanical systems, and with the laser drive replaced by a mechanical drive. Despite the concept's generality, we concentrate on a specific setup that has recently been realized in [16] and for which experimental parameters have been worked out such that all parameter regimes reported here can be realized using current-state technology [29].

\subsection{Model}

We consider the system depicted in Fig. 1(a), where a highly-reflective dielectric membrane is placed in the middle between two high-finesse mirrors.In contrast to the standard setup, comprising one mode inside an optical cavity whose frequency changes due to the mechanical displacement of a movable end-mirror, here the membrane's displacement $x$ affects several modes, ones that are confined to the left and others to the right half of the cavity. We consider two modes $a_{L}, a_{R}$ to the left and right of the membrane, respectively. Due to the membrane's partial transparency, photons can tunnel through the device leading to an optical coupling between $a_{L}$ and $a_{R}$. The system Hamiltonian reads

$$
\hat{H}_{\text {sys }}=\hbar \omega_{0}\left(1-\frac{x(t)}{l}\right) \hat{a}_{L}^{\dagger} \hat{a}_{L}+\hbar \omega_{0}\left(1+\frac{x(t)}{l}\right) \hat{a}_{R}^{\dagger} \hat{a}_{R}+\hbar g\left(\hat{a}_{L}^{\dagger} \hat{a}_{R}+\hat{a}_{R}^{\dagger} \hat{a}_{L}\right)+\hat{H}_{\text {drive }}+\hat{H}_{\text {decay }}
$$

Here, $\hat{a}_{L}^{\dagger} \hat{a}_{L}$ and $\hat{a}_{R}^{\dagger} \hat{a}_{R}$ are the number operators for the left and right mode, respectively, $l$ denotes the length of both cavity halves and $g$ represents the optical coupling frequency between the two modes. The displacement of the membrane $x$ linearly changes the bare modes' frequency $\omega_{0}$. While for a given position $x$ the frequency of one optical mode is increased, the one of the other mode is decreased and vice versa. Due to the coupling there is an avoided crossing in the optical resonance frequency $\omega_{ \pm}(x)= \pm \sqrt{g^{2}+\left(\omega_{0} x / l\right)^{2}}$, see Fig. $1(\mathrm{~b}) . \hat{H}_{\text {drive }}$ and $\hat{H}_{\text {decay }}$ denote terms that need to be added to describe laser driving and photon decay out of the cavity.

We assume the membrane to be driven with mechanical frequency $\Omega$ around a mean position $x_{0}$ with amplitude $A$ such that the displacement reads

$$
x(t)=A \cos (\Omega t)+x_{0}
$$

The dynamics of the system will be particularly interesting if the timescale of the mechanical motion at frequency $\Omega$ is comparable to the timescale of photon exchange between the two modes, described in terms of the coupling frequency $g$; i.e. $g \simeq \Omega$. In this case the photon dynamics can no longer be considered as quasi static and non-equilibrium photon dynamics must be taken into account. In particular, for $g \simeq \Omega$ the excitation spectrum of the cavity will no longer resemble the two hyperbola branches depicted in Fig. 1(b). Furthermore, the mechanics can start to shuttle photons between the two modes of the cavity. We point out that the optical coupling frequency $g$ in such a setup has recently been significantly 

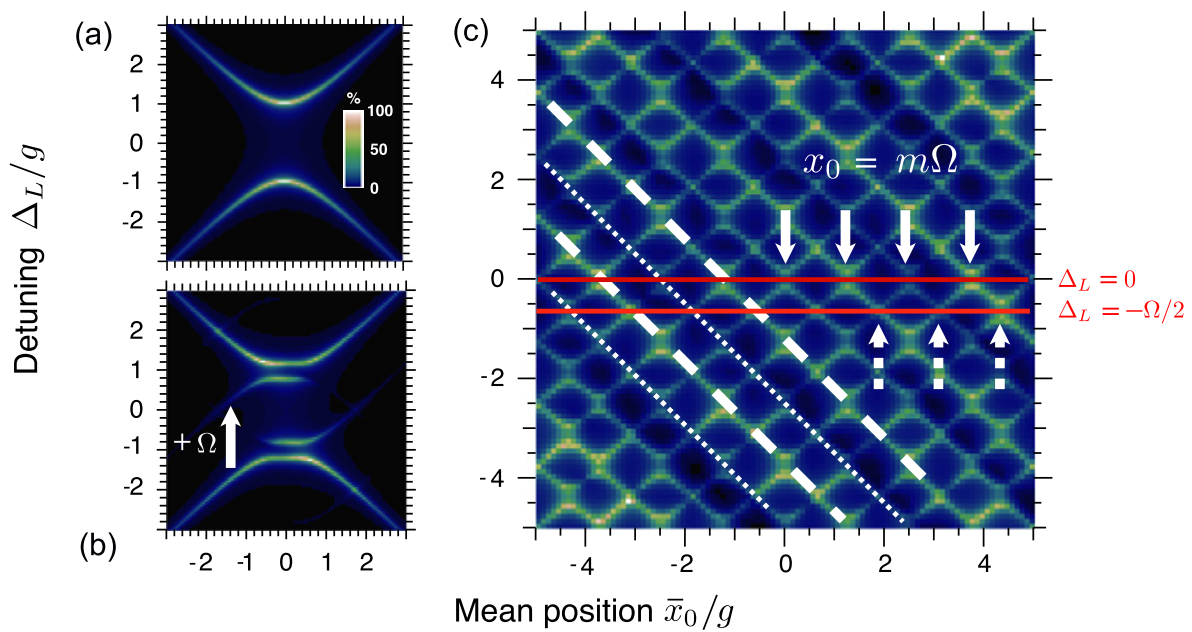

Fig. 2. Transmission spectrum: density plot of the time-averaged transmission to the right, depending on the membrane's mean position $\bar{x}_{0}$ and the laser detuning $\Delta_{L}$. (a) For no mechanical driving $(\bar{A}=0)$ the spectrum corresponds to the hyperbola branches depicted in Fig. 1(b). (b) For modest driving ( $\bar{A} / \Omega=0.1, \Omega=2 \mathrm{~g}$ ) mechanical sidebands appear. When the mechanical frequency $\Omega$ matches the optical modes' frequency difference, we can observe Autler-Townes splittings and high transmission due to mechanically driven Rabi dynamics. (c) For strong driving $(\bar{A} / \Omega=10, \Omega / 2 \pi=0.2 \mathrm{~g})$ the spectrum comprises many sidebands while the original splitting is renormalized. The strength of, and the size of the splitting between the individual mechanical excitations reveal details on the photon dynamics. For instance Landau-Zener-Stueckelberg oscillations in the light field dynamics manifest themselves in a modulation of the splitting sizes for a change in drive amplitude $\bar{A}$. Solid (dashed) arrows indicate $\bar{x}_{0}=m \Omega+\Delta_{L}$ for $\Delta_{L}=0\left(\Delta_{L}=-\Omega / 2\right.$ ).

reduced and is tunable down to $200 \mathrm{kHz}$ [18]. Thus, for mechanical frequencies in the MHz range, $g \simeq \Omega$ is experimentally accessible. Note that this requires membranes with very high reflectivity. Beyond optical setups, the regime $g \simeq \Omega$ is particularly relevant for coupled multimode microwave setups where the coupling frequencies between microwave modes are naturally in the regime of typical mechanical frequencies [32]. Mechanically driven coherent photon dynamics, for instance, will enable new ways to manipulate microwave fields in terms of mechanical driving.

It turns out, the simplest way to investigate this dynamics in an experiment is to measure the transmission. We assume the left hand side of the cavity to be driven by an external laser at frequency $\omega_{L}$ and investigate the transmission $T(t)$ to the right. The cavity decay is considered via a constant cavity decay rate $\kappa$ for each of the optical modes $a_{L}, a_{R}$. In the following we will always consider the laser frequency $\omega_{L}$ with respect to the bare modes' frequency $\omega_{0}$ and define the laser detuning $\Delta_{L}=\omega_{L}-\omega_{0}$. To abbreviate the notation we will furthermore express all lengths in terms of frequency: $\bar{A}=\left(\omega_{0} / l\right) A$, $\bar{x}_{0}=\left(\omega_{0} / l\right) x_{0}$. We will consider two distinct parameter regimes, see Fig. 1(c), i.e. weak/modest and strong mechanical driving. Note that for the latter regime with $\bar{A} \gg \bar{x}_{0}$, we repeatedly sweep through the avoided crossing (Fig. 1(b), (c)).

\subsection{Transmission spectrum}

Given the Hamiltonian, we calculate the transmission to the right using input-output theory $T(t)=\kappa\left\langle\hat{a}_{R}^{\dagger}(t) \hat{a}_{R}(t)\right\rangle /\left(b^{i n}\right)^{2}$, where $b^{\text {in }}$ is the amplitude of the laser drive. The result can be expressed as

$$
T(t)=\kappa^{2}\left|\int_{-\infty}^{t} G\left(t, t^{\prime}\right) e^{-i \Delta_{L} t^{\prime}-(\kappa / 2)\left(t-t^{\prime}\right)} \mathrm{d} t^{\prime}\right|^{2}
$$

see [29]. The exponential of the integrand includes the laser drive at $\Delta_{L}=\omega_{L}-\omega_{0}$ and the cavity decay rate $\kappa$, while the Green's function $G\left(t, t^{\prime}\right)$ describes the amplitude for a photon to enter the laser-driven left mode at time $t^{\prime}$ and to be found in the right mode later at a time $t$. The explicit form of $G\left(t, t^{\prime}\right)$ will be stated below.

Fig. 2 shows numerical results on the transmission spectrum. Without mechanical driving, Fig. 2(a), the transmission corresponds to the optical resonance frequency depicted in Fig. 1(b). For modest driving, Fig. 2(b), mechanical sidebands appear whose intensity depend on the driving strength, i.e. the amplitude $\bar{A}$. In particular, when the mechanical frequency $\Omega$ matches the frequency difference between the two optical modes we observe Autler-Townes splittings and high transmission indicating mechanically driven Rabi dynamics. This mechanically assisted process shuttles photons between the left and the right mode. For strong driving, Fig. 2(c), many mechanical sidebands appear. The details on the sidebands' intensity as well as the size of the additional avoided crossings between them comprise a wealth of information on the underlying photon dynamics that we will discuss in the following. 


\subsection{Physical description: Multiphonon transition picture}

To understand the dynamics, we note that the observation of transmission through the cavity will be determined by two subsequent processes. First, the laser drive must excite the left mode $a_{L}$ to insert photons into the cavity. Second, the internal dynamics must be able to transfer photons from the left into the right mode such that transmission can actually be observed. In general, both processes are inelastic and require energy to be transferred between the light fields and the oscillating membrane. In the following we will restrict ourselves to the regime of strong mechanical driving $\bar{A} \gg g, \Omega$.

To excite the cavity, the laser at $\Delta_{L}$ has to be in resonance with the left mode's frequency, $-\bar{x}(t)=-\bar{x}_{0}+\bar{A} \cos (\Omega t)$, that is oscillating around its mean frequency $-\bar{x}_{0}$. Apart from the obvious resonance at $\Delta_{L}=-\bar{x}_{0}$, the spectrum of a periodically modulated mode comprises mechanical sideband due to excitations in terms of multiphonon transitions. Thus, the condition to excite the cavity and to insert a photon into the left mode reads

$$
\Delta_{L}+m \Omega=-\bar{x}_{0}
$$

see Fig. 1(b), where $m \Omega$ is an adequate multiple of the mechanical frequency $\Omega$. The width of the individual resonance is determined by the cavity decay rate $\kappa$. Note that due to the strong driving the effective coupling between the two modes is very small such that it does not contribute to the resonance condition.

The dynamics of the second process, i.e. the internal dynamics transferring photons between the left and the right half of the cavity, can be mapped to the dynamics of a two-state system, represented by the two optical modes that are driven by the mechanics [29]. For strong mechanical driving, this turns out to be analogous two a two-state system that is periodically swept through an avoided crossing where LZ-transitions might occur [33,34]. In our case, an LZ transition splits the photon state into a coherent superposition, the two amplitudes gather different phases and interfere the next time the system transverses the avoided crossing. For two-state systems, the resulting interference pattern in the state population is known as Stueckelberg oscillations [35]. This pattern depends on the details such as the driving strength and driving frequency. The same process might as well be depicted as a second multiphonon transition from the left mode with average frequency $-\bar{x}_{0}$ (where photons enter the cavity due to the laser drive) to the right one at $+\bar{x}_{0}$, see Fig. 1 (b). Thus, the second resonance condition becomes apparent,

$$
n \Omega=2 \bar{x}_{0}
$$

\subsection{Landau-Zener-Stueckelberg oscillations in the photon dynamics}

The multiphonon transition picture allows us to understand the essential processes involved as well as the overall structure of the resonances occurring in Fig. 2(c). However, the resonance conditions Eqs. (3) and (4) are insufficient to understand the details of the spectrum as well as to identify the fingerprints of two- and multilevel dynamics within the light field. To accomplish this we have to take a closer look at the details of the dynamics.

Analyzing the equation of motion for the system described by the Hamiltonian (1) yields that the Green's function $G\left(t, t^{\prime}\right)$ in Eq. (2) can be factorized $G\left(t, t^{\prime}\right)=\tilde{a}_{R}\left(t, t^{\prime}\right) e^{-i \phi\left(t^{\prime}\right)}$, where $\phi\left(t^{\prime}\right)=(\bar{A} / \Omega) \sin \left(\Omega t^{\prime}\right)$ and $\tilde{a}_{R}\left(t, t^{\prime}\right)$ is a solution to

$$
i \frac{\mathrm{d}}{\mathrm{d} t}\left(\begin{array}{c}
\tilde{a}_{R} \\
\tilde{a}_{L}
\end{array}\right)=\left(\begin{array}{cc}
\bar{x}_{0} & g e^{+2 i \phi(t)} \\
g e^{-2 i \phi(t)} & -\bar{x}_{0}
\end{array}\right)\left(\begin{array}{c}
\tilde{a}_{R} \\
\tilde{a}_{L}
\end{array}\right)
$$

with $t \geqslant t^{\prime}$ and initial condition $\tilde{a}_{R}\left(t^{\prime}, t^{\prime}\right)=0, \tilde{a}_{L}\left(t^{\prime}, t^{\prime}\right)=1$. Each factor describes one of the processes discussed above [29]. For the excitation,

$$
e^{-i(\bar{A} / \Omega) \sin \left(\Omega t^{\prime}\right)}=\sum_{m} J_{m}(\bar{A} / \Omega) e^{-i m \Omega t^{\prime}}
$$

describes possible multiphonon transitions $m \Omega$ in addition to excitations due to the laser at $\Delta_{L}$, see (3). The strength of the individual process is determined by a Bessel function $J_{m}(\bar{A} / \Omega)$. Similarly, the strength of the second multiphonon transition $n \Omega$ (see Eq. (4)), originating from the internal photon dynamics mapped to a two-state system described by $\tilde{a}_{R}\left(t, t^{\prime}\right)$, turns out to be determined by $J_{n}(2 \bar{A} / \Omega)$. This second process, that is necessary to transfer photons from the left into the right mode, and its dependence on amplitude $\bar{A}$ involves the physics of Landau-Zener-Stueckelberg oscillations. While the strength of the individual mechanical sidebands in Fig. 2(c) depend on $J_{m}(\bar{A} / \Omega)$, the splittings between them are governed by $J_{n}(2 \bar{A} / \Omega)$. Note that this sensitively depend on the indices for the Bessel functions ( $m, n$ ) according to Eqs. (3) and (4).

Given these insights, an approximate expression for the time-averaged transmission (2) can be worked out, see [29],

$$
T=\left(\frac{\kappa}{g}\right)^{2} \sum_{m}\left\{J_{m}\left(\frac{\bar{A}}{\Omega}\right) \frac{J_{n}\left(2 \frac{\bar{A}}{\Omega}\right)}{\frac{1}{g^{2}}\left[\left(\frac{\kappa}{2}\right)^{2}+\left(\bar{x}_{0}+\left[m-\frac{1}{2}\right] \Omega\right)^{2}\right]+\left[J_{n}\left(2 \frac{\bar{A}}{\Omega}\right)\right]^{2}}\right\}_{n=2 m-1}^{2}
$$

displayed here for the special case of $\Delta_{L}=-\Omega / 2$, see indication in Fig. 2(c). To derive this formula we assumed the setup to be in the resolved sideband regime $(\Omega>\kappa)$. Fig. 3(a) shows the result.According to the multiphonon transition picture, 

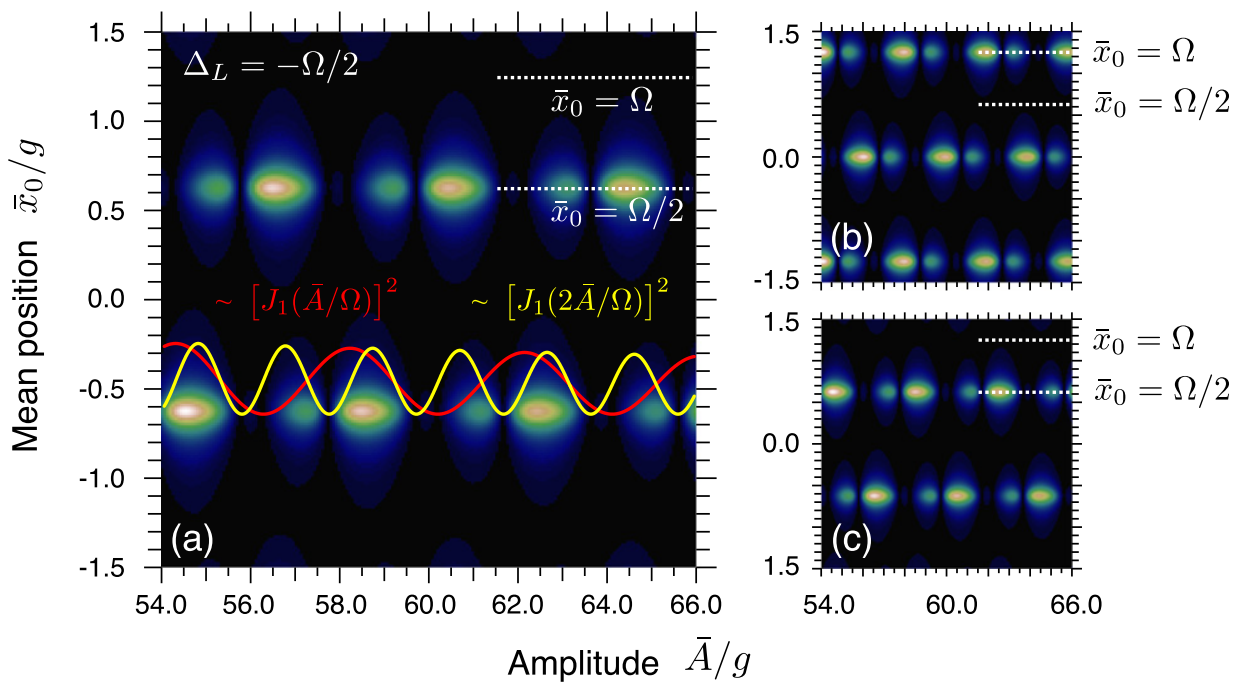

Fig. 3. (a) Density plot for the time-averaged transmission according to the analytic expression (6) dependent on average displacement $\bar{x}_{0}$ and drive amplitude $\bar{A} \gg \Omega, g$ (parameters: laser detuning $\Delta_{L}=-\Omega / 2$, mechanical frequency $\Omega / 2 \pi=0.2 g$ and cavity decay rate $\kappa=0.2 g$ ). Transmission can only be observed if both resonance conditions Eqs. (3) and (4) are fulfilled. The resonance width is set by $\kappa$. As a function of amplitude $\bar{A}$, the transmission is modulated according to the product of two Bessel functions. For $\bar{x}_{0}=-\Omega / 2$ both are depicted in the plot's plane; red: $\sim J_{m}^{2}(\bar{A} / \Omega)$ due to excitation process; yellow: $\sim J_{n}^{2}(2 \bar{A} / \Omega)$, due to Landau-Zener-Stueckelberg dynamics. (b) Analytic results for identical parameters but $\Delta_{L}=0$. (c) $\Delta_{L}=+\Omega / 2$.

(a)

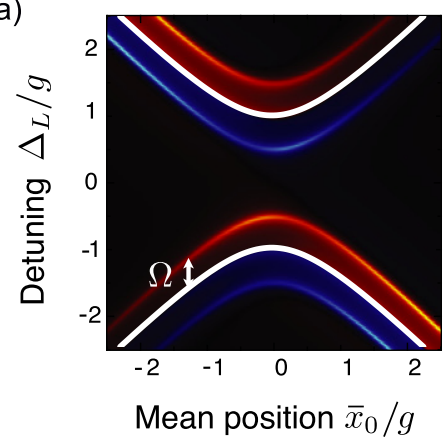

(b)

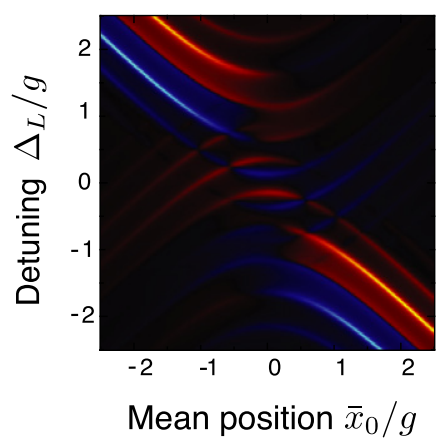

Fig. 4. Density plot of the effective optomechanical damping/heating rate $\Gamma_{\text {opt }}$ versus mean position $\bar{x}_{0}$ and laser detuning $\Delta_{L}$ for the system depicted in Fig. 1(a) (see [17] and [37]). Multiple mechanical sidebands occur for increasing amplitude (a) $\bar{A} / \Omega=0.001$, (b) $\bar{A} / \Omega=1.5$. Due to multiphonon transitions, a laser detuning of $m \Omega$ above or $-m \Omega$ below the optical hybridized modes' frequency gives rise to heating ( $\Gamma_{\text {opt }}<0$, red) or cooling ( $\Gamma_{\text {opt }}>0$, blue) of the membrane's mechanical motion, respectively. Further parameters are optical coupling $g=2 \Omega$ and cavity decay rate $\kappa / \Omega=0.2$.

transmission can only be observed if both resonance conditions (3) and (4) are met. Furthermore, in the case of resonance, the transmission is modulated by the product of the two Bessel functions introduced above. While $J_{m}(\bar{A} / \Omega)$ is due to the excitation process, $J_{n}(2 \bar{A} / \Omega)$ is the results of the internal dynamics showing Landau-Zener-Stueckelberg oscillation in the light field dynamics of the mechanically driven optomechanical system. In Fig. 2(c) these oscillations as function of amplitude $\bar{A}$ would appear as modulation of the splitting size between individual sidebands for fixed detuning $\Delta_{L}$.

If we increase the laser frequency from $\Delta_{L}=-\Omega / 2$ (Fig. 3(a)), we first tune out of resonance until we hit the subsequent ones at $\Delta_{L}=0$ (Fig. 3(b)) and $\Delta_{L}=+\Omega / 2$ (Fig. 3(c)). Note that the transmission patterns change as a different set of Bessel functions $J_{m}, J_{n}$ is involved, according to $(m, n)$ in (3) and (4). Finally note that the analytic result plotted in Fig. 3(b) very well agrees with numerical results shown in [29] for identical parameters.

\subsection{Outlook: Mechanically driven photon dynamics}

Beyond the dynamics of Landau-Zener-Stueckelberg oscillations, mechanically driven coherent photon dynamics in principle allows to realize all kinds of driven two- and multilevel dynamics, known from quantum optics, in the light field of optomechanical systems. For several mechanical and optical modes, for instance, one may think of analogues to driven atomic $\Lambda$ and $V$-type systems and effects such as coherent trapping or electromagnetically induced transparency. Multimode optomechanical systems may furthermore exhibit modified optomechanical cooling and heating properties. Fig. 4 illustrates the effective optomechanical damping and heating rate for the setup in Fig. 1(a). The mechanically driven photon 

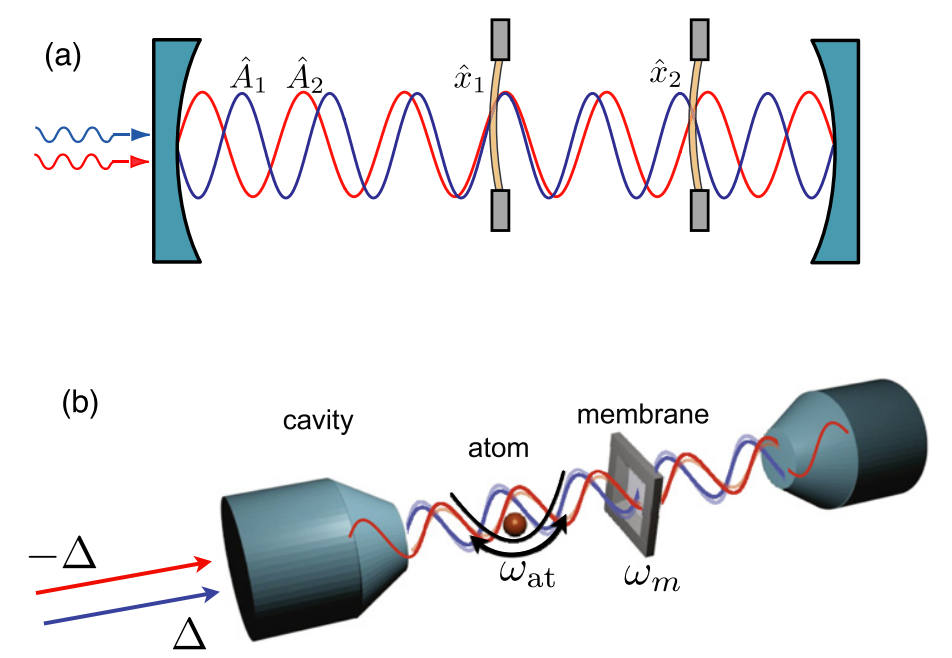

Fig. 5. (a) Two mechanical oscillators $\left(\hat{x}_{1,2}\right)$ are coupled to the fields $\left(\hat{A}_{1,2}\right)$ of an optical cavity. The cavity fields can induce an effective coupling between the mechanical oscillators. This scheme can be employed to achieve strong coupling between a mechanical membrane and the motion of a single atom trapped in the cavity fields (b).

dynamics discussed above will thus be essential to understand self-induced nonlinear optomechanical oscillations as well as dynamical multistability [36], applied to the new domain of multimode optomechanical systems [37].

\section{Dynamics of two mechanical oscillators inside an optical cavity}

In this section, we consider setups that comprise two mechanical modes in an essential way. Elimination of the light field then yields an effective interaction between the modes. This might be useful to couple the motion of a single atom to that of a membrane, among other applications. We briefly review that particular atom-membrane example, which has been presented recently in more detail elsewhere [30,31].

\subsection{Model}

As another straight-forward extension of the standard optomechanical setup (with only one mechanical and one optical mode), we now turn toward a system consisting of two mechanical oscillators $\left(\hat{x}_{1}, \hat{x}_{2}\right)$ that couple to two field modes $\left(\hat{A}_{1}, \hat{A}_{2}\right)$ of a driven, optical cavity, cf. Fig. 5 . This system is described by a Hamiltonian

$$
\begin{aligned}
\hat{H} & =\hbar \sum_{i=1,2} \omega_{i} \hat{b}_{i}^{\dagger} \hat{b}_{i}-\hbar \sum_{j=1,2}\left(\Delta_{j}-\sum_{i=1,2} \tilde{g}_{i j}\left(\hat{b}_{i}^{\dagger}+\hat{b}_{i}\right)\right) \hat{A}_{j}^{\dagger} \hat{A}_{j}+\hat{H}_{\text {drive }}+\hat{H}_{\text {decay }} \\
& =\hat{H}_{0}+\hat{H}_{\text {drive }}+\hat{H}_{\text {decay }}
\end{aligned}
$$

where the cavity fields are transformed into the frame rotating at the driving frequency $\omega_{L, j}$, and $\Delta_{j}=\omega_{L, j}-\omega_{0, j}$ denotes the detuning parameter with respect to the resonance frequency of the corresponding cavity mode, $\omega_{0, j}$. $\hat{A}_{1,2}$ and $\hat{b}_{1,2}$ are the annihilation operators of the optical field modes and the mechanical oscillators respectively. The mechanical oscillation frequencies are given by $\omega_{i}$, and

$$
\hat{x}_{i}=\sqrt{\hbar / 2 m_{i} \omega_{i}}\left(\hat{b}_{i}^{\dagger}+\hat{b}_{i}\right)=\ell_{i}\left(\hat{b}_{i}^{\dagger}+\hat{b}_{i}\right)
$$

represents the position operator of the $i$ th oscillator. The optomechanical interaction is induced by the radiation pressure force that is proportional to the light field intensities and leads to a coupling rate between the $j$ th optical and the $i$ th mechanical mode denoted by $\tilde{g}_{i j}$. $\hat{H}_{\text {drive }}$ and $\hat{H}_{\text {decay }}$ take account of the laser driving and the decay via cavity losses (at rates $\kappa_{j}$ ) and mechanical damping (at rates $\Gamma_{i}$ ).

The nonlinear dynamics that originate from the intensity-proportional radiation pressure force in optomechanical systems is an interesting field on its own and has been studied for example in $[36,15,38]$. Here, however, we linearize the interaction term around the steady state by introducing $\hat{A}_{j}=\alpha_{j}+\hat{a}_{j}$, where $\alpha_{j}$ is the classical field amplitude and $\hat{a}_{j}$ takes account of the quantum fluctuations around the classical value, which are considered to first order in $\hat{a}_{j}$ only. As a result the Hamiltonian (7) reduces to

$$
\hat{H}=\hbar \sum_{i=1,2} \omega_{i} \hat{b}_{i}^{\dagger} \hat{b}_{i}-\hbar \sum_{j=1,2} \Delta_{j} \hat{a}_{j}^{\dagger} \hat{a}_{j}+\sum_{i, j=1,2} g_{i j}\left(\hat{b}_{i}^{\dagger}+\hat{b}_{i}\right)\left(\hat{a}_{j}^{\dagger}+\hat{a}_{j}\right)+\hat{H}_{\text {decay }}+\text { const. }
$$

where we defined $g_{i j}=\tilde{g}_{i j} \alpha_{j}$. 


\subsection{Cavity-induced coupling between two mechanical oscillators}

The interaction of the oscillators with the cavity fields can lead to an effective coupling between the oscillators. To arrive at a simple expression for the coupling rate we restrict ourselves to the symmetric case of equally strong light fields $\left(\alpha_{1}=\alpha_{2}=\alpha\right)$ whose laser detunings are chosen equal in magnitude but with opposite $\operatorname{sign}\left(\Delta_{1}=-\Delta_{2}=\Delta\right)$. Moreover we assume that the optomechanical coupling rates are equal in strength with $g_{11}=g_{12}=g_{1}$ and $-g_{21}=g_{22}=g_{2}$. Note that these conditions can be matched by a suitable choice of the corresponding system parameters, namely the cavity length, the laser frequency and the position of the oscillators inside the cavity, as depicted in Fig. 5 and discussed in detail in [30]. Moreover we assume the oscillator eigenfrequencies to be equal $\left(\omega_{1}=\omega_{2}=\omega\right)$ in order to achieve maximal coupling strength. The general scheme of the coupling mechanism, however, does not rely on this particular choice of the parameters.

Integrating out the cavity degrees of freedom leads to a Hamiltonian describing two harmonic oscillators that are linearly coupled:

$$
\hat{H}=\hbar \sum_{i=1,2} \omega \hat{b}_{i}^{\dagger} \hat{b}_{i}-\hbar G\left(\hat{b}_{1}^{\dagger}+\hat{b}_{1}\right)\left(\hat{b}_{2}^{\dagger}+\hat{b}_{2}\right)+\hat{H}_{\text {decay }}
$$

where the effective coupling strength is given by

$$
G=\frac{2 g_{1} g_{2}(\Delta-\omega)}{(\Delta-\omega)^{2}+\kappa^{2} / 4}+\frac{2 g_{1} g_{2}(\Delta+\omega)}{(\Delta+\omega)^{2}+\kappa^{2} / 4}
$$

A detailed derivation of this coupling term in a description based on a Lindblad master equation for the system's density matrix can be found in [31]. The analysis also introduces an expression for the decoherence rate that describes the dissipative part of the interaction between the oscillators and the cavity:

$$
\Gamma_{c}^{ \pm}=\frac{\kappa\left(g_{1}^{2}+g_{2}^{2}\right)}{(\Delta \pm \omega)^{2}+\kappa^{2} / 4}
$$

In a Lindblad master equation the rates $\Gamma_{c}^{ \pm}$apply to collective modes of the two oscillators [31]. In order to have the coherent interaction dominating over the incoherent one $\left(G / \Gamma_{c}^{ \pm} \gg 1\right)$, the cavity modes are to be driven off-resonant $(\Delta \gg \omega, \kappa)$ and the optomechanical coupling rates are to be chosen equal $\left(g_{1}=g_{2}=g\right)$. In this case the coupling rate can be approximated as $G \simeq 4 g^{2} / \Delta$ such that $G / \Gamma_{c}^{ \pm} \simeq 2 \Delta / \kappa$. This implies that for a far off resonant laser drive the coherent interaction induced via the cavity field can be much stronger than the accompanying decoherence due to photon losses.

The coherent part of the interaction between the oscillators can also be understood by considering virtual transitions that involve the creation or annihilation of a cavity photon. Such transitions allow a phonon to be transferred from one oscillator to the other at a rate that is proportional to the product of the oscillator-cavity couplings $\left(g_{1} g_{2}\right)$ and suppressed by the energy cost $\Delta$ of the virtual transitions. The dissipation rates responsible for the incoherent interaction, $\Gamma_{c}^{ \pm}$, are well-known in the context of optomechanical cooling. They can be combined to represent the optomechanical cooling rate $\Gamma_{\mathrm{opt}}=\Gamma_{c}^{+}-\Gamma_{c}^{-}[39,40]$.

\subsection{Strong coupling between a mechanical resonator and a single atom}

As proposed in [30] the cavity-induced coupling mechanism allows to reach the regime of strong coupling between a nanomechanical membrane $\left(\hat{x}_{1}\right)$ and a single atom $\left(\hat{x}_{2}\right)$. In general any direct coupling between the motion of a single atom and a macroscopic mechanical oscillator is a very challenging task because the coupling rate would typically involve a factor given by $\sqrt{m_{2} / m_{1}} \sim 10^{-7}-10^{-4}$, where $m_{2}$ and $m_{1}$ are the masses of the atom and the macroscopic oscillator. In our setup (Fig. 5(b)), however, where atom and mechanical oscillator couple strongly to a cavity, the cavity-induced interaction can become so strong as to overcome the relevant dissipation rates and to get into the strong coupling regime.

The motion of a nanomechanical membrane couples via radiation pressure to the field of a driven optical cavity in the way described by the Hamiltonian (7) (as has been shown and experimentally verified in [16]).

The dipole force acting on the atom allows both to trap the atom in the field of the two cavity modes and to induce a linear interaction between the motion of the atom and the individual cavity fields. To see this we consider the optical potential seen by the atom:

$$
V\left(\hat{x}_{\mathrm{at}}\right)=\hbar U_{0}\left(\sin ^{2}\left(k_{1} \hat{x}_{2}\right) \hat{A}_{1}^{\dagger} \hat{A}_{1}+\sin ^{2}\left(k_{2} \hat{x}_{2}\right) \hat{A}_{2}^{\dagger} \hat{A}_{2}\right)=V_{1}\left(\hat{x}_{2}\right)+V_{2}\left(\hat{x}_{2}\right)
$$

Here $k_{1}$ and $k_{2}$ denote the wavenumbers of the cavity fields, and $\hat{x}_{2}$ is the position of the atom. The maximal potential energy per photon, given by $\hbar U_{0}$, depends on the detuning of the laser frequency with respect to the atomic transition frequency, on the mode volume, the beam shape inside the cavity and the atomic wave function in the transverse direction. Here it is assumed to be negative and equal for both cavity fields. The atom will be trapped in one of the potential minima at a mean position $\bar{x}_{2}$. An expansion of $V\left(\hat{x}_{2}\right)$ around $\bar{x}_{2}$ yields a quadratic trapping potential in the vicinity of $\bar{x}_{2}$ given by $m_{2} \omega_{2}^{2}=2 \hbar U_{0} \alpha^{2} u^{\prime \prime}\left(\bar{x}_{2}\right)$, where $\alpha$ denotes the steady state amplitude for the cavity fields, and $u(x)=\sin ^{2}\left(k_{1} x\right)+\sin ^{2}\left(k_{2} x\right)$. 
Therefore, at the equilibrium position $\bar{x}_{2}$, the motion of the atom couples linearly to each of the individual cavity fields $\hat{A}_{1}$ and $\hat{A}_{2}$, for nonzero $V_{i}^{\prime}\left(\bar{x}_{2}\right)$. In particular, a positioning of the atom inside the cavity as sketched in Fig. 5(a) (where the second membrane is now replaced by a single atom) allows for $V_{1}^{\prime}\left(\bar{x}_{2}\right)=-V_{2}^{\prime}\left(\bar{x}_{2}\right)$. In this case, the coupling of the atomic motion to the cavity modes is described by rates of equal magnitude and different sign: $g_{21}=-g_{22}=-g_{2}=$ $U_{0} \alpha k_{1} \ell_{2} \sin \left(2 k_{1} \bar{x}_{2}\right)$.

As a result, the system consisting of a mechanical membrane and a single atom, both of which couple to two modes of a driven optical cavity, is described by the Hamiltonian (8) with $\hat{x}_{2}-\bar{x}_{2}=\ell_{2}\left(\hat{b}_{2}^{\dagger}+\hat{b}_{2}\right)$. The system's parameters $U_{0}, m_{1,2}, k_{1,2}$, $\alpha^{2}$ and the equilibrium positions of membrane and atom can be tuned such that $\omega_{1}=\omega_{2}$ and $g_{1}=g_{2}$ [31]. When driving the cavity modes strong enough, the optomechanical coupling rates $g_{1,2} \propto \alpha$ can be made sufficiently large to allow for a large cavity-induced interaction strength $G$ between membrane and atomic motion.

By definition, strong coupling regime is reached when the coherent coupling exceeds all relevant decoherence rates. As already discussed, the interaction with the cavity leads to collective decay rates of the reduced system given by $\Gamma_{c}^{ \pm}$. For $\Delta \gg \kappa$ one can achieve $G / \Gamma_{c}^{ \pm} \gg 1$. Spontaneous emission from the atom's excited state leads to a momentum diffusion due to photon recoil at a rate $\Gamma_{\text {at }}$ which increases with the cavity field intensity, the atom-photon coupling $U_{0}$ and the Lamb-Dicke parameter $k_{1,2} \ell_{2}$ [30]. The membrane on the other hand is subject to thermal fluctuations due to its coupling to the environment leading to a ground state decoherence rate of $\Gamma_{m} \simeq k_{B} T / \hbar Q$, where $T$ is the temperature of the thermal heat bath and $Q$ is the mechanical quality factor. In this context an additional aspect has to be considered: The heating of the membrane by photon absorption sets another limit on the power circulating inside the cavity. In total an optimization of the system's parameters [30,31] shows that the regime of strong coupling, i.e. $G \gg \Gamma_{c}^{ \pm}, \Gamma_{\text {at }}, \Gamma_{m}$ is within reach for nearfuture experiments. In particular, $G / \Gamma_{a t} \gg 1$ essentially requires a large cooperativity for the coupling of the single atom to the cavity, as was demonstrated in CQED experiments [41], while $g / \Gamma_{m}$ requires a large thermal link of the membrane to its support in order to dissipate absorbed laser power sufficiently fast.

\subsection{Applications and outlook}

The cavity-induced coupling scheme presented above offers the opportunity to realize strong coupling between two oscillators even in the case of a very unequal mass ratio. Coherent evolution between a single atom and a nanomechanical membrane would allow to manipulate and control the state of a macroscopic oscillator. As envisaged in [31] the membrane can be brought into a nonclassical state (squeezed state, Fock state) by transferring such a state from the initially prepared atom. Another specific quantum mechanical feature to aim at is the entanglement between the membrane and the atomic motion (see also [42]). We note however that the decoherence mechanisms, in particular the thermal fluctuations acting on the membrane, affect and weaken the coherent evolution severely.

When pursuing the idea of observing finite entanglement in such a system, two routes seem viable. On the one hand one can slightly modify the above setup and impose a temporal modulation of the coupling $\operatorname{constant} G(t)=G \cos ^{2}\left(\frac{\omega_{1}+\omega_{2}}{2} t\right)$ (see [31] for details). This realizes a two-mode squeezing interaction between the atom and the membrane. In this case, for the parameters proposed in [30] it is possible to generate entanglement that is more robust against thermal decoherence than in the original setup. Still this scheme cannot be used to generate entanglement in the steady state as the system is driven into an instable regime and the atomic phonon occupation increases exponentially.

Alternatively, one may let the existing interaction do its job, which would lead to entanglement in the ground state of the coupled system. Then, the challenge is to drive the system into the ground state. For a setup of high optical quality, in particular when the resolved sideband regime $\left(\kappa \ll \omega_{1}\right)$ is reached, it is possible to cool the membrane to the ground state $[39,40]$. In view of the proposed setup of Fig. 5(b) this would require an additional cavity field that is red-detuned to the first sideband $\left(\Delta=-\omega_{1}\right)$. As shown in [43] the optomechanical cooling process can help to generate entanglement between two coupled mechanical oscillators. On the other hand, entanglement is destroyed by the dissipative cooling process when the coupling to the cooling beam becomes too large. The theory presented in [43] allows to identify an optimal coupling strength to the cavity's cooling beam.

The above discussion has shown two of the various possibilities offered by the optomechanical coupling. It can be used to mediate coherent dynamics in hybrid optomechanical systems with very unequal mass ratios. On the other hand, the coupling to cavity fields can be used to generate entanglement between two mechanical oscillators, as has been discussed for the case of two identical membranes in [44] and for the case of a single atom interacting with a mechanical membrane in [31]. These two examples might turn out to be essential tools in future experiments that aim at observing and manipulating the quantum dynamics of multimode, hybrid optomechanical systems.

\section{Conclusions}

Multimode optomechanical setups are just beginning to be explored. They open a large area in which to exploit the interactions between light and mechanics, for fundamental physics and possible applications. Inducing interactions between different mechanical modes (via the light field) or coherent coupling between different optical modes (via mechanical driving) are just two of the simpler examples that we discussed here. 


\section{Acknowledgements}

We acknowledge fruitful collaborations on these topics with Jack Harris (driven photon dynamics), as well as with M. Wallquist, C. Genes, P. Zoller, P. Treutlein, J. Kimble, and J. Ye (atom-membrane coupling). Useful discussions with J. Sankey, S. Girvin, and S. Kohler are also acknowledged.

This research has been supported by the DFG (NIM, SFB 631, Emmy-Noether program), by DIP and GIF, as well as by EU

IP AQUTE (K.H.) and the Chinese Scholarship Council (H.Z.W.).

This research has been supported by the DFG (NIM, SFB 631, Emmy-Noether program), by DIP and GIF, as well as by EU IP AQUTE and the centre of excellence QUEST (K.H.) and the Chinese Scholarship Council (H.W.).

\section{References}

[1] T.J. Kippenberg, K.J. Vahala, Cavity optomechanics: Back-action at the mesoscale, Science 321 (2008) 1172-1176.

[2] F. Marquardt, S.M. Girvin, Optomechanics, Physics 2 (2009) 40.

[3] C.H. Metzger, K. Karrai, Cavity cooling of a microlever, Nature 432 (2004) 1002-1005.

[4] D. Kleckner, D. Bouwmeester, Sub-kelvin optical cooling of a micromechanical resonator, Nature 444 (2006) 75-78.

[5] S. Gigan, H.R. Böhm, M. Paternostro, F. Blaser, G. Langer, J.B. Hertzberg, K.C. Schwab, D. Bäuerle, M. Aspelmeyer, A. Zeilinger, Self-cooling of a micromirror by radiation pressure, Nature 444 (2006) 67-70.

[6] I. Favero, C. Metzger, S. Camerer, D. König, H. Lorenz, J.P. Kotthaus, K. Karrai, Optical cooling of a micromirror of wavelength size, Appl. Phys. Lett. 90 (2007) 104101.

[7] T. Corbitt, Y. Chen, E. Innerhofer, H. Müller-Ebhardt, D. Ottaway, H. Rehbein, D. Sigg, S. Whitcomb, C. Wipf, N. Mavalvala, An all-optical trap for a gram-scale mirror, Phys. Rev. Lett. 98 (2007) 150802.

[8] T. Carmon, H. Rokhsari, L. Yang, T.J. Kippenberg, K.J. Vahala, Temporal behavior of radiation-pressure-induced vibrations of an optical microcavity phonon mode, Phys. Rev. Lett. 94 (2005) 223902.

[9] A. Schliesser, O. Arcizet, R. Riviere, G. Anetsberger, T.J. Kippenberg, Resolved-sideband cooling and position measurement of a micromechanical oscillator close to the Heisenberg uncertainty limit, Nat. Phys. 5 (2009) 509-514.

[10] G. Anetsberger, O. Arcizet, Q.P. Unterreithmeier, R. Riviere, A. Schliesser, E.M. Weig, J.P. Kotthaus, T.J. Kippenberg, Near-field cavity optomechanics with nanomechanical oscillators, Nat. Phys. 5 (2009) 909-914.

[11] J.D. Teufel, T. Donner, M.A. Castellanos-Beltran, J.W. Harlow, K.W. Lehnert, Nanomechanical motion measured with an imprecision below that at the standard quantum limit, Nat. Nano. 4 (2009) 820-823.

[12] T. Rocheleau, T. Ndukum, C. Macklin, J.B. Hertzberg, A.A. Clerk, K.C. Schwab, Preparation and detection of a mechanical resonator near the ground state of motion, Nature 463 (2010) 72-75.

[13] K.W. Murch, K.L. Moore, S. Gupta, D.M. Stamper-Kurn, Observation of quantum-measurement backaction with an ultracold atomic gas, Nat. Phys. 4 (2008) 561-564.

[14] F. Brennecke, S. Ritter, T. Donner, T. Esslinger, Cavity optomechanics with a Bose-Einstein condensate, Science 322 (2008).

[15] C. Metzger, M. Ludwig, C. Neuenhahn, A. Ortlieb, I. Favero, K. Karrai, F. Marquardt, Self-induced oscillations in an optomechanical system driven by bolometric backaction, Phys. Rev. Lett. 101 (2008) 133903.

[16] J.D. Thompson, B.M. Zwickl, A.M. Jayich, F. Marquardt, S.M. Girvin, J.G.E. Harris, Strong dispersive coupling of a high-finesse cavity to a micromechanical membrane, Nature 452 (2008) 72-75.

[17] A.M. Jayich, J.C. Sankey, B.M. Zwickl, C. Yang, J.D. Thompson, S.M. Girvin, A.A. Clerk, F. Marquardt, J.G.E. Harris, Dispersive optomechanics: a membrane inside a cavity, New J. Phys. 10 (2008) 095008.

[18] J.C. Sankey, C. Yang, B.M. Zwickl, A.M. Jayich, J.G.E. Harris, Strong and tunable nonlinear optomechanical coupling in a low-loss system, Nat. Phys. 6 (2010) 707-712.

[19] H. Miao, S. Danilishin, T. Corbitt, Y. Chen, Standard quantum limit for probing mechanical energy quantization, Phys. Rev. Lett. 103 (2009) 100402.

[20] A.A. Clerk, F. Marquardt, J.G.E. Harris, Quantum measurement of phonon shot noise, Phys. Rev. Lett. 104 (2010) 213603.

[21] C. Zhao, L. Ju, H. Miao, S. Gras, Y. Fan, D.G. Blair, Three-mode optoacoustic parametric amplifier: A tool for macroscopic quantum experiments, Phys. Rev. Lett. 102 (2009) 243902.

[22] J.M. Dobrindt, T.J. Kippenberg, Theoretical analysis of mechanical displacement measurement using a multiple cavity mode transducer, Phys. Rev. Lett. 104 (2010) 033901.

[23] I.S. Grudinin, H. Lee, O. Painter, K.J. Vahala, Phonon laser action in a tunable two-level system, Phys. Rev. Lett. 104 (2010) 083901.

[24] M. Eichenfield, J. Chan, R.M. Camacho, K.J. Vahala, O. Painter, Optomechanical crystals, Nature 462 (2009) 78-82.

[25] M. Eichenfield, R. Camacho, J. Chan, K.J. Vahala, O. Painter, A picogram- and nanometre-scale photonic-crystal optomechanical cavity, Nature 459 (2009) $550-555$.

[26] Q. Lin, J. Rosenberg, D. Chang, R. Camacho, M. Eichenfield, K.J. Vahala, O. Painter, Coherent mixing of mechanical excitations in nano-optomechanical structures, Nat. Photonics 4 (2010) 236-242.

[27] A.H. Safavi-Naeini, O.Painter, Design of optomechanical cavities and waveguides on a simultaneous bandgap phononic-photonic crystal slab, Opt. Express 18 (2010) 14926-14943.

[28] M. Li, W.H.P. Pernice, C. Xiong, T. Baehr-Jones, M. Hochberg, H.X. Tang, Harnessing optical forces in integrated photonic circuits, Nature 456 (2008) 480-484.

[29] G. Heinrich, J.G.E. Harris, F. Marquardt, Photon shuttle: Landau-Zener-Stückelberg dynamics in an optomechanical system, Phys. Rev. A 81 (2010) 011801(R).

[30] K. Hammerer, M. Wallquist, C. Genes, M. Ludwig, F. Marquardt, P. Treutlein, P. Zoller, J. Ye, H.J. Kimble, Strong coupling of a mechanical oscillator and a single atom, Phys. Rev. Lett. 103 (2009) 063005.

[31] M. Wallquist, K. Hammerer, P. Zoller, C. Genes, M. Ludwig, F. Marquardt, P. Treutlein, J. Ye, H.J. Kimble, Single-atom cavity qed and optomicromechanics, Phys. Rev. A 81 (2010) 023816.

[32] G. Heinrich, F. Marquardt, Coupled multimode optomechanics in the microwave regime, Europhys. Lett. 93 (2011) 18003; doi:10.1209/0295-5075/ 93/18003.

[33] L.D. Landau, On the theory of transfer of energy at collisions ii, Phys. Z. Sowjetunion 2 (1932) 46-51.

[34] C. Zener, Non-adiabatic crossing of energy levels, Proc. R. Soc. London A 137 (1932) 696.

[35] E. Stückelberg, Theorie der unelastischen stösse zwischen atomen, Helv. Phys. Acta 5 (1932) 369-422.

[36] F. Marquardt, J.G.E. Harris, S.M. Girvin, Dynamical multistability induced by radiation pressure in high-finesse micromechanical optical cavities, Phys. Rev. Lett. 96 (2006) 103901. 
[37] H. Wu, G. Heinrich, F. Marquardt, The effect of Landau-Zener dynamics on phonon lasing, arXiv:1102.1647, 2011; http://arxiv.org/abs/1102.1647.

[38] M. Ludwig, B. Kubala, F. Marquardt, The optomechanical instability in the quantum regime, New J. Phys. 10 (2008) 095013.

[39] F. Marquardt, J.P. Chen, A.A. Clerk, S.M. Girvin, Quantum theory of cavity-assisted sideband cooling of mechanical motion, Phys. Rev. Lett. 99 (2007) 093902.

[40] I. Wilson-Rae, N. Nooshi, W. Zwerger, T.J. Kippenberg, Theory of ground state cooling of a mechanical oscillator using dynamical backaction, Phys. Rev. Lett. 99 (2007) 093901.

[41] R. Miller, T.E. Northup, K.M. Birnbaum, A. Boca, A.D. Boozer, H.J. Kimble, Trapped atoms in cavity qed: coupling quantized light and matter, J. Phys. B 38 (2005) S551-S565.

[42] C. Genes, A. Mari, D. Vitali, P. Tombesi, Quantum effects in optomechanical systems, Adv. At. Mol. Opt. Phys. 57 (2009) 33-86.

[43] M. Ludwig, K. Hammerer, F. Marquardt, Entanglement of mechanical oscillators coupled to a nonequilibrium environment, Phys. Rev. A 82 (2010) 012333; doi:10.1103/PhysRevA.82.012333.

[44] M.J. Hartmann, M.B. Plenio, Steady state entanglement in the mechanical vibrations of two dielectric membranes, Phys. Rev. Lett. 101 (2008) 200503. 